\title{
Mechanical bowel preparation and antimicrobial prophylaxis in elective colorectal surgery in Switzerland-a survey
}

\author{
Adrian Businger • Gabriela Grunder • \\ Marc-Olivier Guenin • Christoph Ackermann • \\ Ralph Peterli • Markus von Flüe
}

Received: 20 August 2009 / Accepted: 19 October 2009 / Published online: 24 October 2010

(C) Springer-Verlag 2010

\begin{abstract}
Background The aim of this study was to assess the use of mechanical bowel preparation (MBP) and antimicrobial prophylaxis in elective colorectal surgery in Switzerland. Methods Ninety-eight heads of surgical departments in Switzerland and 42 visceral surgeons in private practice were asked to answer an 18-item questionnaire in October 2008 about arguments in favor of or against MBP. The participants also indicated whether they use MBP and antimicrobial prophylaxis in colorectal surgery, and if so, what agents were used. Of the participants, $117 / 140(83 \%)$ responded. Additional data were collected pertaining to the respondents' experience and work situation.

Results MBP was used significantly more often for rectal surgery than for left colonic resections $(83 \%$ vs. $53 \%$; $p<$ 0.001 ) and more often for left than for right colonic resections ( $53 \%$ vs. $43 \%$; $p=0.001)$, regardless of the open or laparoscopic approach. Younger surgeons and surgeons
\end{abstract}

Oral presentation at the 96th Meeting of the Swiss Surgical Society, June 2009, Montreux, Switzerland and at the Annual Meeting of the Swiss Society of Gastroenterology and the Swiss Society for Visceral Surgery, September 2009, Zurich, Switzerland

A. Businger $(\bowtie)$

Department of Surgery, Division of Visceral Surgery and

Transplantation, University Hospital Bern,

Bern CH-3010, Switzerland

e-mail: adrian.businger@gmx.ch

G. Grunder

Department of General Surgery and Surgical Research,

University Hospital Basel,

CH-4031 Basel, Switzerland

M.-O. Guenin · C. Ackermann · R. Peterli • M. von Flüe

Department of Surgery,

St. Claraspital,

CH-4016 Basel, Switzerland with a higher case load in colorectal surgery used MBP significantly less frequently in open right colonic resections. For MBP, cathartics were used in $90 \%$ of patients, and enemas were used in $10 \%$ of patients. Of the respondents, $37 \%$ considered MBP to be useful, even very useful. Based on the literature, because of introduction of fast-track protocols or for considerations of patient comfort, $86 \%$ of the respondents had changed the bowel preparation regime during the last 10 years in terms of a reduction of the quantity of cathartics or restricted the indications for MBP. Antimicrobial prophylaxis was used by $100 \%$ of the respondents, $88 \%$ used a single prophylactic dose only, while $70 \%$ administered the antibiotics 30-59 min before the incision. Most of the surgeons used second-generation cephalosporins in combination with metronidazole, and $24 \%$ changed the antibiotic agent or reduced the duration of administration of antibiotics during the last 10 years.

Conclusions MBP is often used in open and laparoscopic rectal surgery, but not in right colonic resections. Scientific evidence regarding MBP has yielded a rethinking about rigorous bowel preparation regimes. As of now, surgeons in Switzerland are not yet unanimously ready to abandon MBP in elective colorectal surgery. In Switzerland, surgeons are influenced by the benefit of antimicrobial prophylaxis in colorectal surgery.

Keywords Mechanical bowel preparation - Antimicrobial prophylaxis · Colorectal surgery - Anastomotic leakage . Septic complications

\section{Introduction}

Mechanical bowel preparation (MBP) is a commonly used regime before elective colorectal surgery. The concept of 
bowel antisepsis and cleansing was described in the late 1960s by Plumley [1] and was continued by several others in the early 1970s [2, 3]. As a result, the use of MBP in colorectal surgery is considered to be a tremendous factor in the prevention of morbidity and mortality due to anastomotic leak and septic complications and has became a conventional expert-based surgical dogma. In point of fact, MBP seems intuitively to have some advantages, such as a decreased intraluminal bacterial count and reduction in troublesome modeled feces, and as a result, simplified bowel handling during the operation and prevention of disruption due to hard feces. The evidence that MBP alone reduces the intraluminal bacterial count [4] or decreases the contamination of the peritoneal cavity during surgery is limited [5]. Indeed, in two updated systematic reviews and meta-analyses, the authors showed no advantages of MBP; the overall rate of anastomotic leakage did not differ significantly between groups with and without MBP $(p=$ 0.46) [6] and (Peto Odds Ratio [OR] $=1.26,95 \%$ confidence interval [CI], 0.941-1.69) [7]. Other authors showed more disadvantages of MBP in colorectal surgery, such as adverse physiologic effects [8] or structural alteration and inflammatory changes in the bowel wall [9]. In contrast, the effectiveness of prophylactic antibiotic prophylaxis in colorectal surgery has been validated [10]. Despite the actual evidence with recent data accumulating against it, MBP is frequently used by general and colorectal surgeons in Western countries. A recommendation to abandon MBP as a routine has not found a place in the guidelines of various surgical societies, as yet. A recent survey of Spanish colorectal surgeons showed a frequency of routinely used MBP of $86 \%$ for laparoscopic surgery and of $87 \%$ for open colorectal surgery [11]. Among 515 US colon and rectal surgeons, the rate of antimicrobial prophylaxis use was $98 \%$, and the rate of MBP use was $99 \%$ [12]. In another survey, of US pediatric surgeons, the usage of antimicrobial prophylaxis and of MBP were similar (99\% and $96 \%$, respectively) for elective colorectal surgery in children [13].

The objective of the present investigation was to assess data on how MBP and antimicrobial prophylaxis are established and rated in the current clinical practices within surgical departments and by surgeons in private practice in Switzerland.

\section{Methods}

Study design

In the autumn of 2008 , a postal survey was sent to the heads of the surgical departments in Switzerland, including five university hospitals (type U clinics), 15 large referral centers (type A clinics), and 83 regional or specialized hospitals (type B clinics), classified according to the Swiss Medical Association [14] and to 42 board-certified surgeons in private practice with a specialist title in visceral surgery. The prospective participants were identified from the 2007 membership files of the Swiss Medical Association, and the surgeons in practice were associated with the Hirslanden private hospital group in Switzerland. After exclusion of two duplicated records and three prospective participants who did not practice colorectal surgery at their institution, 140 surgeons remained eligible for the survey. As the survey addressed healthy people on a voluntary basis without a planned intervention, no further ethical consideration was followed.

\section{Instruments}

The initial questionnaire was developed in German and included 18 items. It was pre-tested for acceptability and clarity among a small group of physicians. Two independent translations in French and Italian were made by professional translators and bilingual physicians, and a final version was obtained by consensus and tested before use. The postal questionnaire consisted of multiple-choice and free-response items. The items addressed the use of and the experience with MBP and the use of antimicrobial prophylaxis in elective colorectal surgery. Additional participant information was extracted from a general form containing questions about hospital category, numbers of surgeons operating on colorectal cases, number of elective resections of the colon and rectum in the year 2007, and the ratio of colorectal surgery on the entire operative volume (activity in colorectal surgery). Demographics were gathered from the membership files of the Swiss Medical Association.

\section{Statistical analysis}

Statistical analysis was performed using standard software, SPSS $^{\circledR} 16$ (SPSS; Chicago, Illinois, USA) and SAS (version 9, SAS, Heidelberg, Germany) for Windows. To compare continuous variables between the two groups, the Mann-Whitney $U$ test was used. Categorical variables were compared using the chi-square test or, when appropriate, the Fisher's exact test. The results are expressed as the mean and standard deviation (SD), unless otherwise stated. All tests were two-sided. A $p$ value $\leq 0.05$ was considered to indicate statistical significance.

\section{Results}

A total of 140 surveys (with a return envelope addressed to the researchers) were sent; after the first mailing and two 
Table 1 Characteristics of the participating institutions

\begin{tabular}{ll}
\hline Characteristic & Value \\
\hline Annual number of colorectal resections & \\
Open colonic & $27(13-69)$ \\
Laparoscopic colonic & $20(8-42)$ \\
Open rectal & $9(4-21)$ \\
Laparoscopic rectal & $2(0-10)$ \\
Number of surgeons for colorectal surgery in $2007^{\mathrm{b}}$ & $4.3(3.0)$ \\
Hospital category & \\
U & 4 \\
A & 13 \\
B3 & 21 \\
B2 & 28 \\
B1 & 15 \\
Practice & 25 \\
Number of beds the day of the survey & $75.4(56.4)$ \\
\hline
\end{tabular}

$U$ university hospitals, $A$ large referral centres, $B 3$ regional or specialized hospitals, $B 2 / 1$ small regional surgical departments (classified according to the Swiss Medical Association [14])

${ }^{\text {a }}$ Year 2007

${ }^{\mathrm{b}}$ Values are expressed as means \pm SD laparoscopic colorectal surgery, no significant differences in MBP use were detected.

Ninety percent of those surgeons who used MBP used cathartics (63\% used polyethylene glycol electrolyte solution (PEG) and 27\% used sodium phosphate), and 10\% used saline enemas; $90 \%$ of the surgeons preferred an ambulatory setting for MBP in elective colorectal surgery. Seven percent of the surgeons replaced electrolytes when they used MBP. Statements in favor of MBP were as follows: makes colonic or rectal resection easier $(38 \%)$, personal experience with MBP (26\%), reduces abdominal contamination $(21 \%)$, reduces septic complications (wound infection and intra=abdominal abscesses; 17\%), and reduces anastomotic leakage $(8 \%)$.

Table 2 Characteristics of the participants

\begin{tabular}{ll}
\hline Characteristic & Value \\
\hline Number & 106 \\
Age (years) ${ }^{\mathrm{a}}$ & $54(41-67)$ \\
Women (\%) & $3(2.8)$ \\
European Board Certification Coloproctology (\%) $^{\text {Experience as surgeon (years) }}{ }^{\mathrm{b}}$ & $7(6.6)$ \\
$\leq 10$ & \\
$>10$ & $15(14.2)$ \\
Activity in colorectal surgery & \\
$<25 \%$ & $91(85.8)$ \\
$26-50 \%$ & \\
$51-75 \%$ & $67(63.2)$ \\
\hline
\end{tabular}

Values in parentheses are percentages unless indicated otherwise

${ }^{a}$ Values are median (range)

${ }^{\mathrm{b}}$ Experience after board certification

${ }^{\mathrm{c}}$ Activity in colorectal surgery $=$ ratio of colorectal surgery on the entire operative volume 
Table 3 Use of mechanical bowel preparation

\begin{tabular}{|c|c|c|c|c|c|c|c|c|c|}
\hline \multirow[t]{2}{*}{ Variable } & \multicolumn{3}{|c|}{ Right colon } & \multicolumn{3}{|l|}{ Left colon } & \multicolumn{3}{|l|}{ Rectum $^{\mathrm{a}}$} \\
\hline & $\begin{array}{l}\text { No MBP } \\
n=60 \\
(\%)\end{array}$ & $\begin{array}{l}\text { MBP } \\
n=46 \\
(\%)\end{array}$ & $p$ & $\begin{array}{l}\text { No MBP } \\
n=50 \\
(\%)\end{array}$ & $\begin{array}{l}\text { MBP } \\
n=56 \\
(\%)\end{array}$ & $p$ & $\begin{array}{l}\text { No MBP } \\
n=17 \\
(\%)\end{array}$ & $\begin{array}{l}\text { MBP } \\
n=82 \\
(\%)\end{array}$ & $p$ \\
\hline Age (years) & & & 0.017 & & & 0.119 & & & 0.595 \\
\hline$<54$ & 70 & 30 & & 51 & 44 & & 20 & 80 & \\
\hline$>54$ & 45 & 55 & & 39 & 61 & & 14 & 86 & \\
\hline Experience of surgeon (years) & & & 0.757 & & & 0.227 & & & 0.098 \\
\hline$<10$ & 67 & 33 & & 67 & 33 & & 36 & 64 & \\
\hline$>10$ & 57 & 43 & & 46 & 54 & & 15 & 85 & \\
\hline Institution & & & 0.131 & & & 0.337 & & & 1.000 \\
\hline University & 100 & 0 & & 75 & 25 & & 0 & 100 & \\
\hline Non-university & 55 & 45 & & 46 & 54 & & 18 & 82 & \\
\hline Activity in colorectal surgery (\%) & & & 0.058 & & & 0.007 & & & 0.588 \\
\hline$<25$ & 47 & 53 & & 37 & 63 & & 20 & 80 & \\
\hline $25-49$ & 69 & 31 & & 66 & 34 & & 15 & 85 & \\
\hline $50-74$ & 100 & 0 & & 33 & 67 & & 0 & 100 & \\
\hline \multicolumn{10}{|l|}{ Colonic resections } \\
\hline Open $^{\mathrm{b}}$ & & & 0.006 & & & 0.079 & & & 1.000 \\
\hline$<27$ & 44 & 56 & & 38 & 62 & & 17 & 83 & \\
\hline$>27$ & 71 & 29 & & 57 & 43 & & 18 & 82 & \\
\hline Laparoscopic & & & 0.043 & & & 0.045 & & & 0.159 \\
\hline$<20$ & 45 & 55 & & 35 & 65 & & 26 & 74 & \\
\hline$>20$ & 67 & 33 & & 56 & 44 & & 11 & 89 & \\
\hline \multicolumn{10}{|l|}{ Rectal resections } \\
\hline Open $^{\mathrm{b}}$ & & & $<0.001$ & & & 0.003 & & & 0.149 \\
\hline$<9$ & 38 & 62 & & 31 & 69 & & 9 & 91 & \\
\hline$>9$ & 73 & 27 & & 61 & 39 & & 20 & 80 & \\
\hline Laparoscopic & & & 0.022 & & & 0.061 & & & 0.773 \\
\hline$<2$ & 39 & 61 & & 31 & 69 & & 17 & 83 & \\
\hline$>2$ & 64 & 36 & & 52 & 48 & & 14 & 86 & \\
\hline
\end{tabular}

MBP mechanical bowel preparation

${ }^{\mathrm{a}}$ Seven missing values

${ }^{\mathrm{b}}$ Year 2007

Of the respondents, $42 \%$ who did not use preoperative MBP prescribed the patients a fluid diet until midnight the evening before surgery, and $22 \%$ let the patients fast; $13 \%$ of surgeons utilized no bowel preparation whatsoever before surgery. The top three ranked statements against MBP given by surgeons who have abandoned and do not use MBP were as follows: a lack of clinical evidence (62\%), personal experience not using MBP (22\%), and patient discomfort (7\%). Of the respondents, $37 \%$ considered MBP to be useful, even very useful, whereas $29 \%$ of surgeons considered MBP to be useless, with $19 \%$ in doubt about the benefit of MBP.

The bowel preparation regime was changed by $86 \%$ of the respondents during the last 10 years, largely because of clinical evidence concerning MBP $(55 \%)$ or because of the introduction of fast-track surgery in their institutions
$(15 \%)$. Of the respondents, $12 \%$ indicated that they will change or change again the current regime (abandonment of MBP, introduction of fast-track surgery, or decrease the amount of cathartics).

Antimicrobial prophylaxis

All surgeons indicated that they routinely use parenteral antimicrobial prophylaxis in elective colorectal surgery. None of the respondents administered antibiotics orally, $88 \%$ used single-dose prophylaxis, and $9 \%$ preferred extended prophylaxis for $48 \mathrm{~h}$. The most-used antibiotics were secondgeneration cephalosporins ( $82 \%$ ) and amoxicillin/clavulanate potassium $(10 \%)$, most often given in combination with metronidazole $(82 \%)$ or ornidazole $(11 \%)$. The first dose of antibiotic was administere $30-59 \mathrm{~min}$ before incision by $70 \%$ 
of the surgeons, and during the last $30 \mathrm{~min}$ before incision by $21 \% ; 9 \%$ of surgeons indicated an interval of 60-120 min prior to surgery. Statements regarding antimicrobial prophylaxis were as follows: reduction in wound infections $(77 \%)$ and in other septic complications (34\%). Antimicrobial prophylaxis was considered to be useful, even very useful by $86 \%$ of the respondents; $10 \%$ of the respondents were not sure about the value of antimicrobial prophylaxis. During the last 10 years, $76 \%$ of surgeons did not make any fundamental changes in their antimicrobial prophylaxis regime, while $24 \%$ of the surgeons reduced the dose, shortened the duration of administration, or adjusted the time interval of administration.

\section{Discussion}

In this nationwide survey of general and colorectal surgeons in Switzerland, the use of MBP varied from $43 \%$ to $83 \%$, depending on the type of resection, and the use of antimicrobial prophylaxis was 100\%. Younger surgeons and surgeons reporting a higher case load in open and laparoscopic colonic and rectal resections used MBP significantly less in open right colonic surgery; except for age and the case load in open colonic and laparoscopic rectal resections, a similar difference existed in open left colonic surgery, but not in rectal surgery.

Our results partially support the findings of other studies, which have shown that surgeons with greater activity in colorectal surgery used less MBP [11], that MBP in right colonic resection is used significantly less frequently than in left colonic or rectal resections [11], and that there is a discrepancy both between the subjectively rated usefulness of MBP and the effective use of MBP [12] and between the current evidence and the preoperative bowel preparation practices among surgeons globally [11-13, 15]. The reason for the latter is largely unknown and might be caused by aesthetic and practical factors, especially in laparoscopic surgery. Others considered the absence of a strong recommendation for abandoning MBP in the guidelines of surgical societies as being reflected in surgeons' continued practice of MBP irrespective of geographical site [16]. The comparison of the MBP rates with other studies among colorectal surgeons in Western countries showed that surgeons in Switzerland used MBP less often than do others [11-13, 17] but astonishingly still to a great extent even when there is little evidence to support its use. Even in Switzerland, where the quality of medicine is first class, the surgeons do not read the literature carefully enough or are reluctant to change their practice despite the scientific evidence. The newest evidence concerning MBP may have influenced the lower rates of MBP usage in our survey. In a total of 13 trials with 4,601 patients, Pineda et al. [18] showed that anastomotic leaks and wound infections did not differ significantly between patients who received MBP and those who did not (anastomotic leaks MBP group 4.2\% vs. non-MBP group 3.5\%; Peto $\mathrm{OR}=$ 1.214 , CI 95\%, 0.899-1.64, $p=0.206$, and wound infection MBP group 9.9\% vs. non-MBP group 8.8\%; Peto $\mathrm{OR}=$ 1.156 , CI 95\%, 0.946-1.413, $p=0.155)$. Two recent metaanalyses from Slim et al. [6] and the Cochrane collaborative group [7] are in good accordance with these findings. Moreover, when all surgical site infections were considered, the meta-analysis done by Slim et al. [6] favored no MBP (OR=1.40 (1.05-1.87), $P=0.02)$. Other authors have shown that MBP is associated with spillage of bowel contents during surgery, which may increase wound infections [19], produce an inflammatory response of the bowel wall which might cause on a greater amount of anastomotic leakage [9], and that MBP is distressing for the patient [20]. Surgeons in Switzerland who have not abandoned MBP yet used sodium phosphate less often and PEG more often than did others (27\% and $63 \%$ vs. $63.5 \%$ and $36.5 \%$ used by Spanish surgeons [11], and $47 \%$ and $32 \%$ used by members of the American Society of Colon and Rectal Surgeons [12]), although sodium phosphate seems to be more effective than PEG in cleansing and more favorable in terms of adverse effects [21]. In the recent data accumulation against MBP in colonic resections, the query about the ideal agent in MBP as well as the role of MBP in rectal surgeries still persists [16].

Recent improvements in morbidity and mortality rates associated with colorectal surgery have not resulted from MBP, but from advances in peri-operative care and the introduction of routine antimicrobial prophylaxis [22, 23]. The rate of use of antimicrobial prophylaxis in the current study $(100 \%)$ compared favorably with previously published data [11, 12]; in contrast, the parenteral route of administration of antibiotics in colorectal surgery in Switzerland was preferred (100\%), whereas Spanish and US surgeons use orally administered antibiotics as well in $13.8 \%$ and $86 \%$ of patients, respectively, or in a combination of both. Parenteral administration yielded similar results compared with a combination of parenteral and oral prophylaxis [10]. Furthermore, the vast majority of surgeons used a single dose of antibiotic prophylaxis only (88\%), in accordance with the results of Bratzler et al. [24] and in contrast to Spanish surgeons (43\%). The timing of administration of antibiotics in this study was different from other studies [11, 12] and in good agreement with the evidence reported by Weber et al. [25] who showed that administration of cefuroxime as a prophylactic antibiotic 30-59 min before incision is more effective than administration during the last $30 \mathrm{~min}$.

This study had some limitations. First, although some variables were collected at the level of the surgical unit (e.g., type of clinic and number of beds), we did not use an analytic approach that incorporated a clustered study design because we focused on the individuals (surgeons) rather than the 
clinics. Second, we performed a cross-sectional study, which cannot determine a causal relationship between some of the variables and which precludes evaluation of temporality. Third, the exclusive reliance on self-reported number of cases and professional characteristics raises the issue of measurement error. Fourth, surveys have only a limited level of evidence (level 5; expert opinions) and might not represent the true reality rather than the mood of participants; nevertheless, surveys do have their place in the surgical literature to investigate clinical practice $[11-13,15,17]$. Finally, the surgeons might have self-censored their responses in the fear of deviating from the actual best practice. The high participation rate $(83 \%)$ implies the contrary, demonstrating the participants' confidence in the investigators. Although we cannot completely exclude a participation bias because of differential participation rates among hospital-based surgeons and surgeons in private practice, we believe that our results represent a good estimate of the true use of MBP and antimicrobial prophylaxis in elective colorectal surgery in Switzerland. The surgeons who participated in this study performed approximately 8,300 colorectal resections in Switzerland, indicating $>95 \%$ of all colorectal resections nationwide, according to the Swiss Federal Statistical Office (http://www.bfs.admin.ch/bfs/portal/de/index/themen/14/ 04/01/data/01.Document.104378.xls).

\section{Conclusions}

In summary, the present study demonstrated differences in MBP between surgeons with different case loads in colorectal surgery and showed that in Switzerland, the current evidence concerning MBP and antimicrobial prophylaxis in elective colorectal surgery attracts interest, and surgeons selectively used MBP for different type of resections. Although the current evidence does not support a general use of MBP in colorectal surgery, the personal surgical experience seems to play a pivotal role in the obviation of a higher compliance with the actual evidence. A stronger body of evidence for the role of MBP in rectal surgeries and the ideal agent for MBP in colorectal surgery is needed for an adequate decision-making of its use. Thereafter, recommendation of abandoning MBP as consensus statements might influence the surgeons' habits. The future will show whether surgeons are ready to abandon MBP in elective colorectal surgery as a practice of the past or whether it will continue to hold its dogmatic place.

Acknowledgments The authors thank the surgeons who participated in this study for their valuable information, Raffaele Galli, M.D., Lucerne, Switzerland, for his aid in translation of the questionnaire; Phillip Hendrickson, Ph.D., Basel, Switzerland and Ulrich Guller, M. D. MHS, Bern, Switzerland, for critical reading; and Ulrich Stefenelli (Services-in-statistics, Wurzburg, Germany) for scientific support.
Conflicts of interest None.

Authors' contributions AB, GG, and MOG participated in the study concept and design, and they coordinated and helped to draft the manuscript and discuss the results. $\mathrm{AB}, \mathrm{CA}, \mathrm{RP}$ and MVF analyzed the data and critically revised the manuscript for important intellectual content. $\mathrm{AB}, \mathrm{GG}$ and $\mathrm{MVF}$ obtained administrative, technical and material support. MVF has supervised the study and takes responsibility for the integrity of the data and the accuracy of the data analysis. All authors read and approved the final manuscript.

\section{References}

1. Plumley PF (1966) A simple regime for preparation of colon before large-bowel surgery. Br J Surg 53(5):413-414

2. Barker $K$ et al (1971) The relative significance of preoperative oral antibiotics, mechanical bowel preparation, and preoperative peritoneal contamination in the avoidance of sepsis after radical surgery for ulcerative colitis and Crohn's disease of the large bowel. Br J Surg 58(4):270-273

3. Dunphy JE (1971) Preoperative preparation of the colon and other factors affecting anastomotic healing. Cancer 28(1):181182

4. Reddy BS et al (2007) Randomized clinical trial of effect of synbiotics, neomycin and mechanical bowel preparation on intestinal barrier function in patients undergoing colectomy. Br J Surg 94(5):546-554

5. Fa-Si-Oen PR et al (2005) Effect of mechanical bowel preparation with polyethyleneglycol on bacterial contamination and wound infection in patients undergoing elective open colon surgery. Clin Microbiol Infect 11(2):158-160

6. Slim K et al (2009) Updated systematic review and metaanalysis of randomized clinical trials on the role of mechanical bowel preparation before colorectal surgery. Ann Surg 249 (2):203-209

7. Guenaga KK et al (2009) Mechanical bowel preparation for elective colorectal surgery. Cochrane Database Syst Rev (1): CD001544

8. Holte K et al (2004) Physiologic effects of bowel preparation. Dis Colon Rectum 47(8):1397-1402

9. Bucher P et al (2006) Morphologic alterations associated with mechanical bowel preparation before elective colorectal surgery: a randomized trial. Dis Colon Rectum 49(1):109112

10. Nelson RL et al (2009) Antimicrobial prophylaxis for colorectal surgery. Cochrane Database Syst Rev (1):CD001181

11. Roig JV et al (2009) Mechanical bowel preparation and antibiotic prophylaxis in colorectal surgery: use by and opinions of Spanish surgeons. Colorectal Dis 11(1):44-48

12. Zmora O et al (2003) Trends in preparation for colorectal surgery: survey of the members of the American society of colon and rectal surgeons. Am Surg 69(2):150-154

13. Breckler FD et al (2007) Survey of pediatric surgeons on current practices of bowel preparation for elective colorectal surgery in children. Am J Surg 193(3):315-318

14. Liste der anerkannten Weiterbildungsstätten. Swiss Medical Association (FMH). 2008. Available at http://www.fmh.ch/ shared/data/pdf/wbs_chirurgie.pdf. Accessed 1 July 2008

15. Lassen K et al (2005) Patterns in current perioperative practice: survey of colorectal surgeons in five northern European countries. BMJ 330(7505):1420-1421 
16. Basu S, Shukla VK (2008) Mechanical bowel preparation: are we ready for a paradigm shift? Dig Surg 25(5):325-327

17. Kehlet $\mathrm{H}$ et al (2006) Care after colonic operation-is it evidencebased? Results from a multinational survey in Europe and the United States. J Am Coll Surg 202(1):45-54

18. Pineda CE et al (2008) Mechanical bowel preparation in intestinal surgery: a meta-analysis and review of the literature. J Gastrointest Surg 12(11):2037-2044

19. Mahajna A et al (2005) Bowel preparation is associated with spillage of bowel contents in colorectal surgery. Dis Colon Rectum 48(8):1626-1631

20. Jung B et al (2007) Preoperative mechanical preparation of the colon: the patient's experience. BMC Surg 7:5
21. Itani KM et al (2007) Polyethylene glycol versus sodium phosphate mechanical bowel preparation in elective colorectal surgery. Am J Surg 193(2):190-194

22. Dellinger EP et al (1994) Quality standard for antimicrobial prophylaxis in surgical procedures. Infectious diseases society of America. Clin Infect Dis 18(3):422-427

23. Jimenez JC, Wilson SE (2003) Prophylaxis of infection for elective colorectal surgery. Surg Infect Larchmt 4(3):273-280

24. Bratzler DW et al (2005) Antimicrobial prophylaxis for surgery: an advisory statement from the National Surgical Infection Prevention Project. Am J Surg 189(4):395-404

25. Weber WP et al (2008) The timing of surgical antimicrobial prophylaxis. Ann Surg 247(6):918-926 